\title{
Volume holograms constructed from computer-generated masks
}

\author{
S. K. Case and W. J. Dallas
}

\begin{abstract}
A new type of hologram that combines computer-generated holograms with volume holograms is described. This hologram allows arbitrary selection of the location and color of a computer-generated image when white light illumination is used. Potential applications include optical information processing, holographic optical elements, multicolor displays, and lens testing. Calculations are made to determine the range of wavelengths possible for image reconstruction. Experimental results are given and discussed.
\end{abstract}

\section{Introduction}

Computer-generated holograms (CGH) can be used to generate arbitrary optical wavefronts and to create images of objects that never physically existed.1,2 However, images from these holograms must usually be produced with monochromatic light to avoid chromatic dispersion.

Volume holograms, on the other hand, are capable of reproducing optical wavefronts with great precision and high efficiency but are generally not used for the generation of arbitrary wavefronts. They can, however, be constructed to exhibit good wavelength selectivity and, consequently, produce monochrome images when illuminated by a white light source. ${ }^{3,4}$

These two types of holograms are seen to have complementary properties. By combining a computergenerated hologram and a volume hologram, a new type of hologram, the computer-generated volume hologram (CGVH), can be constructed. The CGVH has advantages inherent to both CGHs and to volume holograms.

\section{CGVH Construction}

Although several techniques might be envisioned to produce a $\mathrm{CGVH},{ }^{5}$ one specific construction method is described here that combines a binary Fraunhofer CGH and a volume holographic reflection grating. The basic reflection grating is produced by exposing a plate of holographic film with collimated laser beams 1 and 2

\footnotetext{
When this work was done both authors were with Virginia Polytechnic Institute and State University, Physics Department, Blacksburg, Virginia 24061; W. J. Dallas is now with Philips GmbH, 2000 Hamburg 54, Federal Republic of Germany.

Received 3 October 1977.

0003-6935/78/0815-2537\$0.50/0.

(C) 1978 Optical Society of America.
}

impinging from opposite sides of the film as in Fig. 1. Before the exposure, a CGH is placed in close contact with one side of the holographic film so that the intensity of one of the exposure beams is modulated in accordance with the transmittance of the CGH mask. After processing the film, the resulting reflection hologram has a transverse spatial modulation that is a replica of the CGH mask and a longitudinal modulation (in depth through the emulsion) corresponding to a reflection grating, as shown in Fig. 2. Thus, the process is equivalent to replacing each of the elementary cells of the original CGH with a tiny reflection grating. Each cell of the resulting reflection hologram can have a high diffraction efficiency and the ability to Bragg diffract (reflect) only a narrowband of wavelengths.

For viewing, the CGVH is illuminated with a beam of spatially coherent collimated light, which can even be white light. The monochromatic light diffracted from the CGVH, as shown in Fig. 2, is then Fourier transformed by a simple lens to produce a single color computer-generated image. Because the CGVH is constructed with plane waves, the Bragg angle of each cell in the hologram is the same. Thus, if white light readout is used, the color of the reconstructed image can be selected by merely rotating the CGVH relative to the readout beam. Unfortunately, the position of the image will rotate as the holographic plate is rotated. Size changes in the image associated with a given readout angle or a wavelength shift between the hologram construction wavelength and the intended readout wavelength can easily be compensated for by an appropriate scaling of the CGH before exposure.

An important feature of the CGVH should be noted. Recall that, at the Bragg angle of a reflection grating, the position (angle) of the diffracted wave can be determined from the readout angle and the tilt of the interference fringes. Once the output angle is determined, the wavelength of the diffracted light can then 


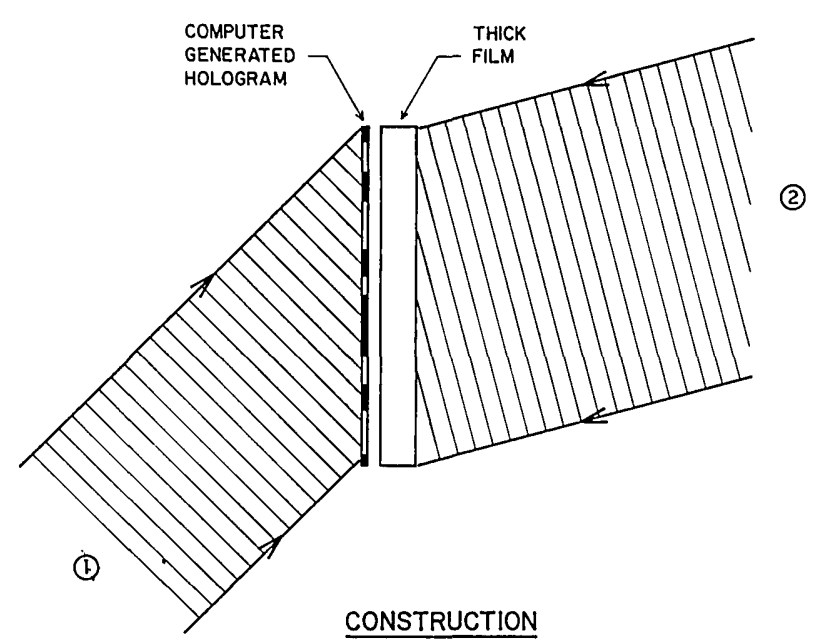

Fig. 1. Construction geometry for a computer-generated volume hologram.

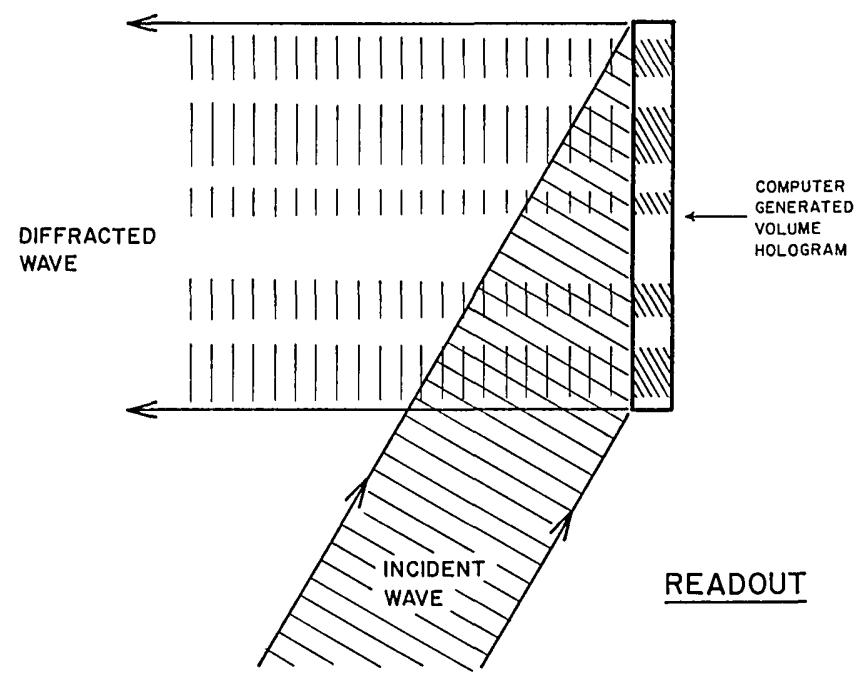

Fig. 2. Readout of a CGVH.

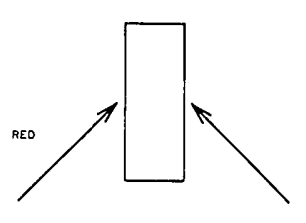

RECORDING (o)

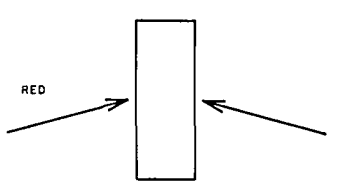

RECORDING (c)

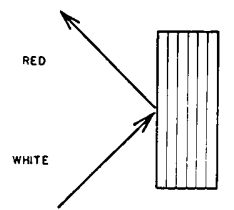

READOUT (b)

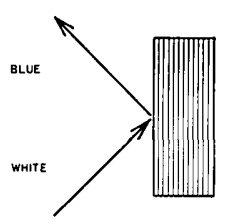

READOUT (d)
Fig. 3. Wavelength selection. Coherent recording beams (a) form a reflection grating that reads out in red (b). The same two recording beams symmetrically rotated to form a different recording geometry (c) construct a reflection grating with smaller grating spacing (d). Readout of this grating at the same incidence angle as in (b) produces a blue diffracted wave (d). The diffracted wavelength can be chosen independently from the diffraction angle.

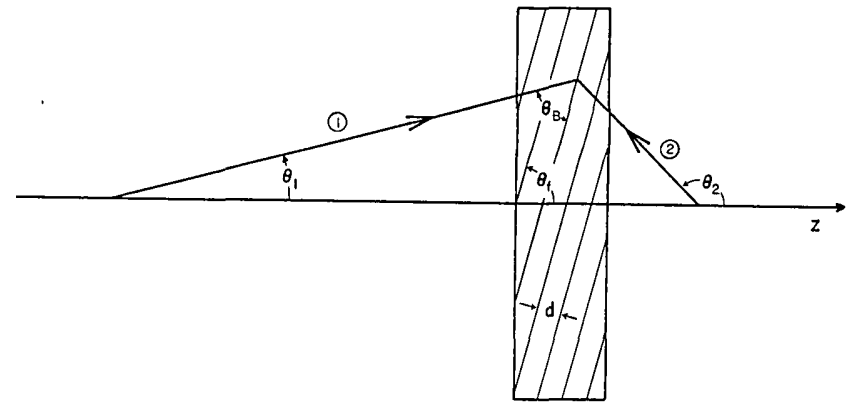

Fig. 4. Construction geometry for a reflection grating. All angles are measured inside the emulsion.

be determined from the spacing between the fringes. Thus, in designing an optical system which uses a CGVH, the position of the image can be specified by the fringe tilt and then the color of the image by the fringe spacing. ${ }^{6}$ By a proper choice of incidence angle for the two laser beams used to construct the CGVH, the tilt and the spacing of the interference fringes within the volume of the hologram can be independently selected. This is possible because the tilt of the fringes depends on the sum of the incidence angles of the exposure beams [see Eq. (1)], while the spacing between the fringes depends on the difference in incidence angles of the two beams [Eq. (3)]. Thus, by properly adjusting the construction beams, the tilt and spacing of the fringes and hence the position and color of the reconstructed image can be independently selected.

As an example, the coherent construction waves in Fig. 3(a) expose a piece of film symmetrically so that the resulting holographic fringes in Fig. 3(b) are parallel to the film surface. Thus, the angle of the incident and diffracted waves in Fig. 3(b) are equal with respect to the film normal. Assume that the readout wave is polychromatic and the light selectively diffracted by the grating is red. Now suppose that it is desired to retain the same readout geometry (incidence and diffraction angles) and yet have the diffracted wave be of shorter wavelength. This is accomplished by constructing another hologram, as shown in Fig. 3(c), with the construction waves symmetrically rotated such that they are both more normal to the film. The resulting holographic fringes will again be parallel to the film plane but will have a smaller spacing. In Fig. 3(d), readout exactly as in Fig. 3(b) will produce a diffracted wave at the same angle as before but with shorter wavelength (blue). Thus, independent control of the position and the color of the diffracted wave is possible.

\section{Analysis}

The image positions and colors obtainable with this $\mathrm{CGVH}$ construction method can be calculated. Figure 4 shows the construction of a reflection grating such as that in each cell of the CGVH. All angles are measured inside the emulsion. The interference fringes bisect the angle between incident beams 1 and 2 so that

$$
\theta_{f}=\left(\theta_{1}+\theta_{2}\right) / 2 \text {. }
$$

Waves 1 and 2 are at the Bragg angles $\theta_{B}$ of the grat- 


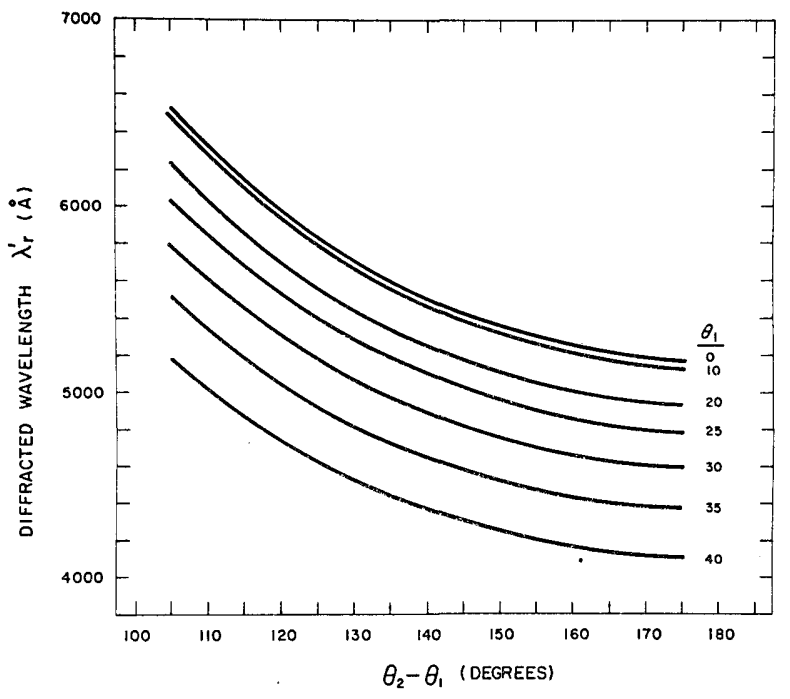

Fig. 5. Diffracted wavelength vs construction parameters. The wavelength $\lambda_{r}^{\prime}$ is measured in free space. The grating construction angles $\theta_{1}$ and $\theta_{2}$ and the readout incidence angle $\theta_{i}$ are measured inside the emulsion. Wavelengths and angles shown are those that can be obtained without the use of prisms for construction and/or readout.

ing that they create and, therefore, obey the Bragg equation

$$
2 d \sin \theta_{B_{\text {construction }}}=\lambda_{c},
$$

where $\lambda_{c}$ is the construction wavelength. The spacing between the fringes is, therefore, given by

$$
d=\frac{\lambda_{c}}{2 \sin \left[\left(\theta_{2}-\theta_{1}\right) / 2\right]} .
$$

Equations (1) and (3) show that the tilt and spacing of the fringes depend on the sum and the difference of the construction angles, respectively.

For readout, broadband spatially coherent collimated illumination at angle $\theta_{i}$ is assumed, where the angle is again measured from the $z$ axis as in Fig. 4. To simplify the analysis, it is assumed that only the wave exactly satisfying the Bragg condition will have appreciable energy and need be considered. To find the wavelength of the diffracted wave $\lambda_{r}$, the Bragg equation

$$
2 d \sin \theta_{B_{\text {readout }}}=\lambda_{r}
$$

must be satisfied. At readout

$$
\theta_{B_{\text {readout }}}=\theta_{f}-\theta_{i}=1 / 2\left(\theta_{1}+\theta_{2}\right)-\theta_{\mathrm{i}} .
$$

Combining Eqs. (3), (4), and (5) yields the diffracted wavelength

$$
\lambda_{r}=\lambda_{c} \frac{\sin \left\{\left[\left(\theta_{1}+\theta_{2}\right) / 2\right]-\theta_{i}\right\}}{\sin \left[\left(\theta_{2}-\theta_{1}\right) / 2\right]} .
$$

Emulsion shrinkage or expansion during film processing must also be accounted for. If the emulsion has thickness $z$ during exposure, it will have thickness $z^{\prime}=$ $\alpha z$ after development. ${ }^{7}$ For $\theta_{f} \approx 90^{\circ}$ and $\alpha=0.82$ (a typical value for Kodak 649-F film with conventional processing in $\mathrm{D}-19)$, the spacing and tilt of the fringes become $d^{\prime} \approx \alpha d$ and $\theta_{f}^{\prime} \approx \theta_{f}$. Therefore, the readout wavelength is modified to yield

$$
\lambda_{r}^{\prime}=\alpha \lambda_{c} \frac{\sin \left\{\left[\left(\theta_{1}+\theta_{2}\right) / 2\right]-\theta_{i}\right\}}{\sin \left[\left(\theta_{2}-\theta_{1}\right) / 2\right]} .
$$

To compensate for emulsion shrinkage or expansion, the fringes can be initially exposed to having a spacing $1 / \alpha \times$ as large as the desired final spacing, or else chemical swelling agents can be used. ${ }^{8}$

The output angle $\theta_{r}$ can be most easily found by considering reflection of the readout wave from the interference fringes. ${ }^{9}$. This gives

$$
\theta_{r}=2 \theta_{f}-\theta_{i}=\theta_{1}+\theta_{2}-\theta_{i} .
$$

Using Eqs. (7) and (8), the output from the CGVH is calculated. Assume $\lambda_{c}=6328 \AA, \alpha=0.82$, and $\theta_{1}+$ $\theta_{2}=185^{\circ}$ so that the fringes are tilted by $2.5^{\circ}$ relative to the film plane (to separate angularly the diffracted image from the specular reflection from the film plate). Figure 5 shows the reconstruction wavelength from the CGVH (measured outside the emulsion) as a function of construction variables. The readout angle $\theta_{i}$ is given as a parameter.

The graph shows that, for a chosen readout angle $\theta_{i}$, a large range of output wavelengths can be achieved by varying the construction parameter $\theta_{2}-\theta_{1}$. Note particularly that, for $\theta_{i} \approx 25^{\circ}$, it is possible to construct the CGVH such that its output could range anywhere from red to blue covering nearly the entire visible spectrum. The graph cari also be used to find the range of output colors available by varying the incidence angle $\theta_{i}$ if the construction parameter $\theta_{2}-\theta_{1}$ is fixed.

Only angles are shown that can be achieved without the use of prisms (or other means) to overcome the critical angle of the film. If prisms are used during the construction and/or readout process such that beams may be incident into the film at internal angles up to $80^{\circ}$ (a practical limit), the calculated readout wavelength ranges from $1125 \AA<\lambda_{r}^{\prime}<23,000 \AA$. Thus computergenerated optical elements can easily be generated for use with ir or other optical systems.

\section{Experimental Results}

A binary $\mathrm{CGH}$ of the letter $O$ was made for use as the construction mask for the CGVH. A CGVH was constructed with $\lambda_{c}=6328 \AA, \theta_{1}=33^{\circ}$, and $\theta_{2}=150^{\circ}$. Xylene or oil is used as an index matching liquid between the $\mathrm{CGH}$ and the Kodak 649-F holographic film.

For readout, the CGVH is illuminated from the substrate side of the film so that emu'sion distortions involved with processing will have a minimal effect on the diffracted wave. ${ }^{10}$ With $\theta_{i}=11^{\circ}$ and white light illumination, the image in Fig. 6 is obtained. The bright area on the left is the specular reflection from the film substrate. The image of the letters is centered $3^{\circ}$ to the right of the specular reflection. The image is slightly above the specular reflection because the hologram construction waves were not precisely coplanar. With the given readout conditions, the letters are orange $\left(\lambda_{r}^{\prime}\right.$ 


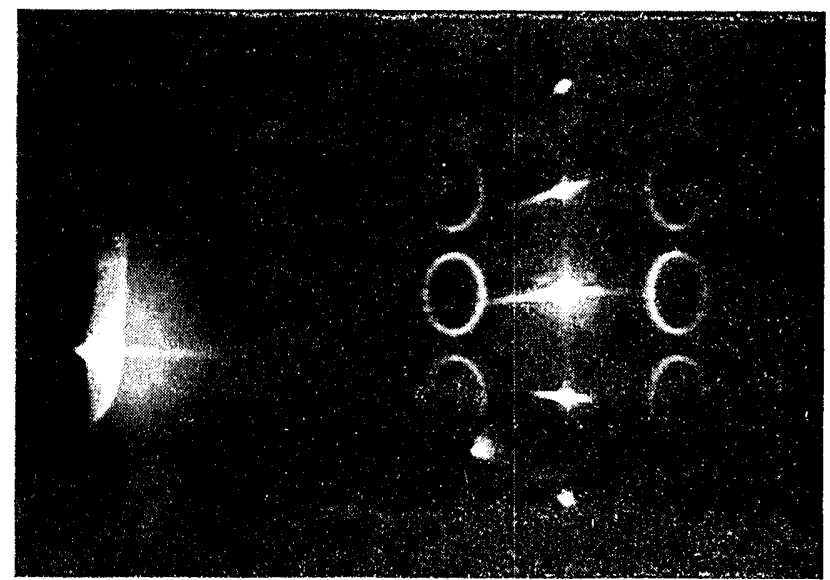

Fig. 6. Monochrome image obtained from a CGVH.

$\approx 6000 \AA$ ). The line between the letters is a dispersed spectrum, which is strongly peaked at $\lambda^{\prime}=6000 \AA$.

Although this CGVH is an absorption reflection hologram and has fairly low diffraction efficiency, constructing the CGVH in a volume phase material such as dichromated gelatin could improve the efficiency up to the level of the original CGH. That is, the elemental cells of the CGVH could diffract $100 \%$ of the desired light just as the open cells of the binary CGH transmit $100 \%$ of the incident light. The curves in Fig. 5 would be quite similar if dichromated gelatin were used, since the exposure wavelength is shorter but the emulsion does not shrink during processing. ${ }^{11}$ Methods, such as that of Lowenthal and Chavel, ${ }^{12}$ to copy only one order of the CGH onto a second hologram might also be adopted to improve the efficiency of the CGVH.

\section{Applications}

With the CGVH, it is possible to produce arbitrarily shaped monochrome wavefronts using temporally incoherent illumination. Because the position and color of a CGVH generated image can be independently selected and the size of the image adjusted by a suitable scaling of the CGH mask, it is also possible to produce multicolor images using only a single color laser to construct the required CGVH. To achieve this, multiple-exposure techniques must be used, and one CGH mask must be prepared for each primary color in the image.
Although the above discussion describes the use of CGVHs to produce images, the technique could also be used to produce color-coded spatial filters for optical information processing. Using this method, for instance, two or more spatial filters could be color multiplexed onto one piece of film. If such a filter were used in an optical pattern recognition system, different objects could be recognized by correlation peaks of different color. The third dimension of the film (depth) is thus used to increase the information storage capacity of the spatial filter.

CGVHs could be useful in the generation of multicolor wavefronts for the testing of aspheric lenses. In addition, the volume hologram construction technique, which allows arbitrary color selection for readout, can be used to record high efficiency holograms for readout at wavelengths for which there are no suitable laser recording sources or photosensitive films.

Partial support from the National Science Foundation is appreciated. The authors are indebted to J. M. Fournier for numerous discussions during the course of the research and preparation of the manuscript. Useful discussions with S. P. Almeida and technical assistance from J. Partin and K. Pritz are also acknowledged.

\section{References}

1. B. R. Brown and A. W. Lohmann, Appl. Opt. 5, 967 (1966).

2. A. W. Lohmann and D. P. Paris, Appl. Opt. 6, 1739 (1967).

3. H. Kogelnik, Bell Syst. Tech. J. 48, 2909 (1969).

4. R. J. Collier, C. B. Burckhardt, and L. H. Lin, Optical Holography (Academic, New York, 1971), Chap. 9.

5. D. R. MacQuigg [Appl. Opt. 16, 1380 (1977)] has demonstrated a technique for recording computer-generated wavefronts into a volume transmission hologram.

6. The wavelength of diffracted light depends on the incidence angle, fringe tilt, and fringe spacing, but it is assumed that the incidence angle and fringe tilt are chosen first so that specifying the fringe spacing will then determine the diffracted wavelength.

7. M. Lehmann, Holography (Focal Press, New York, 1970), p. 72.

8. Ref. 4, p. 288.

9 H. W. Smith, Principles of Holography (Interscience, New York, 1969), p. 66.

10. Ref. 4, p. 519.

11. S. K. Case, "Multiple Exposure Holography in Volume Materials," Thesis, University of Michigan (1976) (University Microfilms, Inc. order no. 76-27, 461), p. 94.

12. S. Lowenthal and P. Chavel, Appl. Opt. 13, 718 (1974). 